\title{
Improved Composite-Boson Theory of Monolayer and Bilayer Quantum Hall Ferromagnets
}

\author{
Z. F. Ezawa \\ Department of Physics, Tohoku University, Sendai 980, Japan
}

May 5, 2018

\begin{abstract}
An improved composite-boson theory of quantum Hall ferromagnets is formulated both for the monolayer and bilayer systems. In this scheme the field operator describes solely the physical degrees of freedom representing the deviation from the ground state. Skyrmions are charged excitations confined to the lowest Landau level. By evaluating the excitation energy of one skyrmion in the interlayer-coherent phase it is shown that the bilayer QH state becomes stabler as the interlayer density difference becomes larger.
\end{abstract}

PACS: 73.40.Hm, 73.20.Dx, 73.40.-c, 75.10.-b

Keywords: quantum Hall effect, quantum coherence, bilayer electron system, skyrmions

\section{Introduction}

The quantum Hall $(\mathrm{QH})$ effect is a remarkable macroscopic quantum phenomenon in the two-dimensional electron system [1]. The underlying physics is understood by the compositeboson $(\mathrm{CB})$ picture [2, 3, 4, 5] or by the composite-fermion picture [6, 7]. There are several new approaches [8, 9] to the problem by extending these pictures.

When the spin degree of freedom is taken into account, a spin coherence develops spontaneously and turns the $\mathrm{QH}$ system into a $\mathrm{QH}$ ferromagnet, provided the Zeeman effect is reasonablly small. Quasiparticles are skyrmions [10], whose existence has been established experimentally [11]. On the other hand, a pseudospin (interlayer) coherence develops spontaneously in certain bilayer QH systems [12, 13, 14]. Some of its characteristic behaviors have already been observed experimentally [15, 16]. In a coherent state the phase and number differences are observables simultaneously. The phase difference is controlled by applying the parallel magnetic field [15] while the number difference is controlled by applying bias voltages to the bilayer system [16].

In this paper we analyze skyrmion excitations in monolayer and bilayer $\mathrm{QH}$ ferromagnets. We use the improved composite-boson (CB) theory [8], proposed based on a suggestion due 
to Girvin [3], Read [4] and Rajaraman et al. [5]. The advantages of the improved scheme are that the field operator describes solely the physical degrees of freedom representing the deviation from the ground state and that the semiclassical property of excitations is determined directly by the microscopic wave function.

In bilayer QH systems we have two types of skyrmions; the spin-skyrmion associated with the spin coherence and the pseudospin-skyrmion associated with the pseudospin coherence. One skyrmion consists of a pair of charged excitations on the two layers, and hence acquires a capacitive charging energy, whichever type of skyrmion it may be. We have predicted [12, 13] that the interlayer-coherent state continues to exist even if the electron densities are made arbitrarily unbalanced between the two quantum wells, as has been confirmed experimentally [16]. Furthermore it has been revealed [16] that the activation energy increases as the density imbalance increases. We explain this characteristic behavior by evaluating the excitation energy of one skyrmion as a function of the density imbalance: The major energy is the capacitive charging energy we mentioned. Throughout the paper we use the natural units $\hbar=c=1$.

\section{Bosonization}

We summarize the idea of the improved CB theory applied to the monolayer spin-frozen $\mathrm{QH}$ state. We denote the electron field by $\psi(\boldsymbol{x})$ and its position by the complex coordinate, which we normalize as $z=(x+i y) / 2 \ell_{B}$ with $\ell_{B}$ the magnetic length. Any state $|\mathfrak{S}\rangle$ at the filling factor $\nu=1 / m$ ( $m$ odd) is represented by the wave function,

$$
\mathfrak{S}[\boldsymbol{x}] \equiv\left\langle 0\left|\psi\left(\boldsymbol{x}_{1}\right) \cdots \psi\left(\boldsymbol{x}_{N}\right)\right| \mathfrak{S}\right\rangle=\omega[z] \mathfrak{S}_{\mathrm{LN}}[\boldsymbol{x}]
$$

where $\mathfrak{S}_{\mathrm{LN}}[\boldsymbol{x}]$ is the Laughlin function,

$$
\mathfrak{S}_{\mathrm{LN}}[\boldsymbol{x}]=\prod_{r<s}\left(z_{r}-z_{s}\right)^{m} \exp \left[-\sum_{r=1}^{N}\left|z_{r}\right|^{2}\right],
$$

and $\omega[z] \equiv \omega\left(z_{1}, z_{2}, \cdots, z_{N}\right)$ is an analytic function symmetric in all $N$ variables. The mapping from the fermionic wave function $\mathfrak{S}[\boldsymbol{x}]$ to the bosonic function $\omega[z]$ defines a bosonization. We call the underlying boson the dressed composite boson and denote its field operator by $\varphi(\boldsymbol{x})$. The field operator turns out to be the one considered first by Read [4] and revived recently by Rajaraman et al. [5]. It follows that

$$
\mathfrak{S}_{\varphi}[\boldsymbol{x}] \equiv\left\langle 0\left|\varphi\left(\boldsymbol{x}_{1}\right) \cdots \varphi\left(\boldsymbol{x}_{N}\right)\right| \mathfrak{S}\right\rangle=\omega[z]
$$

The Laughlin state is represented by $\mathfrak{S}_{\varphi}[\boldsymbol{x}]=1$. A typical quasiparticle (vortex) state [17] is

described by $\mathfrak{S}_{\varphi}[\boldsymbol{x}]=\prod_{r}^{N} z_{r}$, leading to $\langle\varphi(\boldsymbol{x})\rangle=z$ in the semiclassical approximation. This is a highly nontrivial constraint, which turns out to determine all semiclassical properties 
of the vortex excitation. Consequently, the field operator $\varphi(\boldsymbol{x})$ describes solely the physical degrees of freedom representing the deviation from the ground state, and the semiclassical property of excitations is determined directly by the microscopic wave function (11).

We now construct the improved CB theory explicitly. We start with the kinetic Hamiltonian for planar electrons in external magnetic field $(0,0,-B)$,

$$
H_{K}=\frac{1}{2 M} \int d^{2} x \psi^{\dagger}(\boldsymbol{x})\left(P_{x}-i P_{y}\right)\left(P_{x}+i P_{y}\right) \psi(\boldsymbol{x})
$$

where $P_{j}=-i \partial_{j}+e A_{j}^{\text {ext }}$ is the covariant momentum with $A_{j}^{\text {ext }}=\frac{1}{2} \varepsilon_{j k} x_{k} B$. Here, $\varepsilon_{12}=$ $-\varepsilon_{21}=1$ and $\varepsilon_{11}=\varepsilon_{22}=0$. We denote the electron density by $\rho(\boldsymbol{x})=\psi^{\dagger}(\boldsymbol{x}) \psi(\boldsymbol{x})$. The Coulomb term is

$$
H_{C}=\frac{1}{2} \int d^{2} x d^{2} x V(\boldsymbol{x}-\boldsymbol{y}) \varrho(\boldsymbol{x}) \varrho(\boldsymbol{y}),
$$

with $V(\boldsymbol{x})=\left(e^{2} / \varepsilon\right)|\boldsymbol{x}|^{-1}$, where $\varrho(\boldsymbol{x}) \equiv \rho(\boldsymbol{x})-\rho_{0}$ is the density deviation from its average $\rho_{0} \equiv\langle\rho(\boldsymbol{x})\rangle$. It is normalized to vanish, $\left\langle H_{C}\right\rangle=0$, on the homogeneous ground state.

At sufficiently low temperature all relevant excitations are those confined to the lowest Landau level. The condition is that the kinetic energy (卌) is quenched on the state,

$$
\left(P_{x}+i P_{y}\right) \psi(\boldsymbol{x})|\mathfrak{S}\rangle=0
$$

We call it the lowest-Landau-level (LLL) condition.

We define the bare CB field by way of an operator phase transformation of the electron field $\psi(\boldsymbol{x})$,

$$
\phi(\boldsymbol{x})=e^{-i \Theta(\boldsymbol{x})} \psi(\boldsymbol{x})
$$

The phase field $\Theta(\boldsymbol{x})$ is chosen to attach $m$ units of Dirac flux quanta $2 \pi / e$ to each electron via the relation,

$$
\varepsilon_{i j} \partial_{i} \partial_{j} \Theta(\boldsymbol{x})=2 \pi m \rho(\boldsymbol{x}) .
$$

When $m$ is odd, $\phi(\boldsymbol{x})$ is proved to be a bosonic operator. The bare composite boson is the one familiar in literatures [2, 12, 13].

We proceed to define the dressed CB field $\varphi(\boldsymbol{x})$,

$$
\varphi(\boldsymbol{x})=e^{-\mathcal{A}(\boldsymbol{x})} \phi(\boldsymbol{x}) .
$$

Here, the hermitian field $\mathcal{A}(\boldsymbol{x})$ is to be determined so that the basic formula (3) is obtained for the wave function. Substituting (9) and (7) into (4), the kinetic Hamiltonian is transformed into

$$
H_{K}=\frac{1}{2 M} \int d^{2} x \varphi^{\ddagger}(\boldsymbol{x})\left(\mathcal{P}_{x}-i \mathcal{P}_{y}\right)\left(\mathcal{P}_{x}+i \mathcal{P}_{y}\right) \varphi(\boldsymbol{x}),
$$


where $\varphi^{\ddagger}(\boldsymbol{x}) \equiv \varphi^{\dagger}(\boldsymbol{x}) e^{2 \mathcal{A}(\boldsymbol{x})}$, with which $\rho(\boldsymbol{x})=\psi^{\dagger}(\boldsymbol{x}) \psi(\boldsymbol{x})=\varphi^{\ddagger}(\boldsymbol{x}) \varphi(\boldsymbol{x})$. The covariant momentum is

$$
\mathcal{P}_{j}=-i \partial_{j}+e A_{j}^{\mathrm{ext}}(\boldsymbol{x})+\partial_{j} \Theta(\boldsymbol{x})-i \partial_{j} \mathcal{A}(\boldsymbol{x}) .
$$

By a judicious choice of $\mathcal{A}(\boldsymbol{x})$ we are able to bring the LLL condition (6) into a simple form,

$$
\left(\mathcal{P}_{x}+i \mathcal{P}_{y}\right) \varphi(\boldsymbol{x})|\mathfrak{S}\rangle=-\frac{i}{\ell_{B}} \frac{\partial}{\partial z^{*}} \varphi(\boldsymbol{x})|\mathfrak{S}\rangle=0 .
$$

Indeed, when we choose $\partial_{i} \mathcal{A}(\boldsymbol{x})=\varepsilon_{i j}\left[e A_{j}^{\text {ext }}(\boldsymbol{x})+\partial_{j} \Theta(\boldsymbol{x})\right]$, the covariant momentum reads

$$
\mathcal{P}_{j}=-i \partial_{j}-\left(\varepsilon_{j k}+i \delta_{j k}\right) \partial_{k} \mathcal{A}(\boldsymbol{x}),
$$

from which the LLL condition (12) follows trivially. By using (8), the above definition of $\mathcal{A}(\boldsymbol{x})$ leads to

$$
\boldsymbol{\nabla}^{2} \mathcal{A}(\boldsymbol{x})=2 \pi m\left(\rho(\boldsymbol{x})-\frac{e B}{2 \pi m}\right)
$$

It is easy to see that bare composite bosons feel the effective magnetic field $\mathcal{B}_{\text {eff }}=e^{-1} \nabla^{2} \mathcal{A}(\boldsymbol{x})$ and that $\mathcal{B}_{\text {eff }}$ vanishes on the homogeneous ground state where $\langle\rho(\boldsymbol{x})\rangle=\rho_{0}$. It follows that the homogeneous ground state is realized only at the filling factor $\nu \equiv 2 \pi \rho_{0} / e B=1 / m$, where composite bosons undergo bose condensation. Eq.(14) is solved as

$$
\mathcal{A}(\boldsymbol{x})=m \int d^{2} y \ln \left(\frac{|\boldsymbol{x}-\boldsymbol{y}|}{2 \ell_{B}}\right) \varrho(\boldsymbol{y}),
$$

in terms of the density deviation $\varrho(\boldsymbol{x})$ at $\nu=1 / \mathrm{m}$. It is interpreted that the new CB field (9) is obtained by dressing the bare field $\phi(\boldsymbol{x})$ with a cloud of the effective magnetic field generated by $\mathcal{A}(\boldsymbol{x})$, and hence we have called it the dressed field.

Solving the LLL condition (12) we find that the $N$-body wave function $\mathfrak{S}_{\varphi}[\boldsymbol{x}]$ is an analytic function as in (3). It is an easy exercise to derive the relation [5],

$$
\varphi^{\ddagger}\left(\boldsymbol{x}_{1}\right) \cdots \varphi^{\ddagger}\left(\boldsymbol{x}_{N}\right)|0\rangle=\mathfrak{S}_{\mathrm{LN}}[\boldsymbol{x}] \psi^{\dagger}\left(\boldsymbol{x}_{1}\right) \cdots \psi^{\dagger}\left(\boldsymbol{x}_{N}\right)|0\rangle .
$$

Because of this relation the function $\omega[z]$ in the wave function (11) is given precisely by the formula (3).

One might question the hermiticity of the theory [5], since the covariant momentum (13) has an unusual expression. Analyzing the Lagrangian density we find that the canonical conjugate of $\varphi(\boldsymbol{x})$ is not $i \varphi^{\dagger}(\boldsymbol{x})$ but $i \varphi^{\ddagger}(\boldsymbol{x}) \equiv i \varphi^{\dagger}(\boldsymbol{x}) e^{2 \mathcal{A}(\boldsymbol{x})}$. It implies that the hermiticity is defined together with the measure $e^{2 \mathcal{A}(\boldsymbol{x})}$. Such a measure has arisen since the transformation (9) is not unitary. The covariant momentum (13) is hermitian together with this measure. It is instructive to rewrite the kinetic Hamiltonian (10) as

$$
H_{K}=\frac{\omega_{c}}{2} \int d^{2} x\left(\frac{\partial}{\partial z^{*}} \varphi(\boldsymbol{x})\right)^{\dagger} e^{2 \mathcal{A}(\boldsymbol{x})} \frac{\partial}{\partial z^{*}} \varphi(\boldsymbol{x}),
$$

which is manifestly hermitian. 


\section{Monolayer QH Ferromagnets}

We analyze the QH system with the spin degree of freedom. The electron field $\psi^{\alpha}(\boldsymbol{x})$ is labeled by the spin index $\alpha=\uparrow, \downarrow$. Bare and dressed CB fields $\phi^{\alpha}(\boldsymbol{x})$ and $\varphi^{\alpha}(\boldsymbol{x})$ are defined by (7) and (9), where $\Theta(\boldsymbol{x})$ and $\mathcal{A}(\boldsymbol{x})$ are defined by (8) and (15) with $\rho(\boldsymbol{x})=$ $\Psi^{\dagger}(\boldsymbol{x}) \Psi(\boldsymbol{x})=\Phi^{\dagger}(\boldsymbol{x}) e^{2 \mathcal{A}(\boldsymbol{x})} \Phi(\boldsymbol{x})$. Here, $\Psi(\boldsymbol{x})$ and $\Phi(\boldsymbol{x})$ are two-component fields made of $\psi^{\alpha}(\boldsymbol{x})$ and $\varphi^{\alpha}(\boldsymbol{x})$. We decompose the bare CB field into the U(1) field $\phi(\boldsymbol{x})$ and the $\mathrm{SU}(2)$ field $n^{\alpha}(\boldsymbol{x}), \phi^{\alpha}(\boldsymbol{x})=\phi(\boldsymbol{x}) n^{\alpha}(\boldsymbol{x})$, by requiring $\sum_{\alpha} n^{\alpha \dagger} n^{\alpha}=1$. Thus,

$$
\varphi^{\alpha}(\boldsymbol{x})=e^{-\mathcal{A}(\boldsymbol{x})} \phi^{\alpha}(\boldsymbol{x})=e^{-\mathcal{A}(\boldsymbol{x})} \phi(\boldsymbol{x}) n^{\alpha}(\boldsymbol{x}) .
$$

The field $n^{\alpha}(\boldsymbol{x})$ is the complex-projective field [18], whose overall phase has been removed and given to the $\mathrm{U}(1)$ field $\phi(\boldsymbol{x})$.

The kinetic Hamiltonian reads

$$
H_{K}=\frac{1}{2 M} \int d^{2} x \Phi^{\ddagger}(\boldsymbol{x})\left(\mathcal{P}_{x}-i \mathcal{P}_{y}\right)\left(\mathcal{P}_{x}+i \mathcal{P}_{y}\right) \Phi(\boldsymbol{x}) .
$$

The LLL condition is

$$
\left(\mathcal{P}_{x}+i \mathcal{P}_{y}\right) \Phi(\boldsymbol{x})|\mathfrak{S}\rangle=-\frac{i}{\ell_{B}} \frac{\partial}{\partial z^{*}} \Phi(\boldsymbol{x})|\mathfrak{S}\rangle=0
$$

Therefore the CB wave function $\mathfrak{S}_{\varphi}[z]$ is symmetric and analytic in $N$ coordinates $z_{i}$, with which the electron wave function is given by $\mathfrak{S}[\boldsymbol{x}]=\mathfrak{S}_{\varphi}[z] \mathfrak{S}_{\mathrm{LN}}[\boldsymbol{x}]$.

We use the index $a=x, y, z$ for the spin component. The spin density is

$$
S^{a}(\boldsymbol{x})=\frac{1}{2} \Psi^{\dagger}(\boldsymbol{x}) \tau^{a} \Psi(\boldsymbol{x})=\frac{1}{2} \rho(\boldsymbol{x}) \boldsymbol{s}^{a}(\boldsymbol{x}),
$$

where $\tau^{a}$ is the Pauli matrix and $\boldsymbol{s}^{a}(\boldsymbol{x})$ is the normalized spin field, or the nonlinear sigma field,

$$
s^{a}(\boldsymbol{x})=\boldsymbol{n}^{\dagger}(\boldsymbol{x}) \tau^{a} \boldsymbol{n}(\boldsymbol{x})
$$

with $\boldsymbol{n}(\boldsymbol{x})$ the two-component field made of $n^{\uparrow}(\boldsymbol{x})$ and $n^{\downarrow}(\boldsymbol{x})$. The Zeeman term is

$$
H_{Z}=-\frac{1}{2} g^{*} \mu_{B} B \int d^{2} x \rho(\boldsymbol{x}) s^{z}(\boldsymbol{x})
$$

where $g^{*}$ is the gyromagnetic factor and $\mu_{B}$ the Bohr magneton. Each Landau level contains two energy levels for spin-up and spin-down states with the one-particle gap energy $g^{*} \mu_{B} B$. The ground state $\left|g_{0}\right\rangle$ is unique, whose wave function is

$$
\mathfrak{S}_{\mathrm{g}}^{\mathrm{spin}}[\boldsymbol{x}]=\prod_{r}\left(\begin{array}{l}
1 \\
0
\end{array}\right)_{r}^{\mathrm{spin}} \mathfrak{S}_{\mathrm{LN}}[\boldsymbol{x}]
$$


where the two-component spinor is common to all electrons, representing the spin-up polarization.

The effective Hamiltonian governing perturbative fluctuations of the sigma field has been derived previously [13, 14],

$$
H_{\mathrm{eff}}=\frac{1}{2} \rho_{s} \int d^{2} x\left\{\left[\partial_{k} \boldsymbol{s}(\boldsymbol{x})\right]^{2}-\frac{\rho_{0}}{\rho_{s}} g^{*} \mu_{B} B s^{z}(\boldsymbol{x})\right\} .
$$

The first term represents the spin stiffness with $\rho_{s}=\nu e^{2} /\left(16 \sqrt{2 \pi} \varepsilon \ell_{B}\right)$. Perturbative excitations are charge neutral. We consider the vanishing limit of the Zeeman term $\left(g^{*}=0\right)$. In this case the ground state is given by an arbitrary constant sigma field, $\boldsymbol{s}(\boldsymbol{x})=$ constant. All spins are polarized into one arbitrary direction. There exists a degeneracy in the ground states. The choice of a ground state implies a spontaneous magnetization, or a QH ferromagnetism. When a continuous symmetry is spontaneously broken, there arises a gapless mode known as the Goldstone mode. Quantum coherence develops spontaneously. Actually, due to the Zeeman term all spins are polarized into the $z$ axis. As far as the Zeeman effect is small enough, the system is still considered as a $\mathrm{QH}$ ferromagnet with a finite coherent length.

\section{Topological Excitations}

We analyze topological excitations on the QH ferromagnet. The semiclassical analysis is powerful when the $\mathrm{CB}$ wave function $\mathfrak{S}_{\varphi}[z]$ is factorizable,

$$
\mathfrak{S}^{\operatorname{spin}}[\boldsymbol{x}]=\prod_{r}\left(\begin{array}{c}
\omega^{\uparrow}\left(z_{r}\right) \\
\omega^{\downarrow}\left(z_{r}\right)
\end{array}\right)_{r}^{\mathrm{spin}} \mathfrak{S}_{\mathrm{LN}}[\boldsymbol{x}] .
$$

In this case the one-point function is given by $\left\langle\varphi^{\alpha}(\boldsymbol{x})\right\rangle \equiv \omega^{\alpha}(z)$. Based on the formula (17) it is parametrized as

$$
e^{-\mathcal{A}(\boldsymbol{x})} e^{i \chi(\boldsymbol{x})} \sqrt{\rho_{0}+\varrho(\boldsymbol{x})} n^{\alpha}(\boldsymbol{x})=\omega^{\alpha}(z)
$$

since $|\phi(\boldsymbol{x})|^{2}=\rho_{0}+\varrho(\boldsymbol{x})$. Here and hereafter all fields are classical fields. The CauchyRiemann equation for (26) yields [13],

$$
\varrho(\boldsymbol{x})=-\nu Q_{0}(\boldsymbol{x})+\frac{\nu}{4 \pi} \nabla^{2} \ln \left(1+\frac{\varrho(\boldsymbol{x})}{\rho_{0}}\right),
$$

where use was made of (14), and $Q_{0}(\boldsymbol{x})$ is the topological charge density whose explicit form is given later. A nontrivial density deviation $\varrho(\boldsymbol{x})$ is induced around a topological soliton according to this equation, which we name the soliton equation. Since the soliton equation is a direct consequence of the semiclassical LLL condition (26), it is interpreted that the 
density deviation occurs in order to confine topological excitations within the lowest Landau level.

The excitation carries a quantized electric charge,

$$
q_{e}=-e \int d^{2} x \varrho(\boldsymbol{x})=e \nu \int d^{2} x Q_{0}(\boldsymbol{x})=\nu q e,
$$

as follows from (27), where $q=\int d^{2} x Q_{0}(\boldsymbol{x})$ is the topological charge. We explicitly consider

solitons with $q=1$. One soliton carries a fractional charge, say, $q_{e}=\frac{1}{3} e$ at $\nu=1 / 3$, as has been experimentally observed [19]. We now show that there are topological solitons, vortices and skyrmions, associated with the $\mathrm{U}(1)$ and $\mathrm{SU}(2)$ parts of the CB field $\Phi(\boldsymbol{x})$.

\subsection{Vortex Excitations}

The $\mathrm{U}(1)$ excitation is generated on the ground state when $\partial_{k} \chi(\boldsymbol{x}) \neq 0$ and $n^{\alpha}(\boldsymbol{x})=$ constant in (26). The complex-projective field is trivial, $n^{\uparrow}=1$ and $n^{\downarrow}=0$, representing a spin-up polarized ground state (23). It follows from (26) that the topological charge density is

$$
Q_{0}(\boldsymbol{x}) \equiv Q_{0}^{V}(\boldsymbol{x})=\frac{1}{2 \pi} \varepsilon_{j k} \partial_{j} \partial_{k} \chi(\boldsymbol{x}),
$$

as represents the vorticity.

The simplest excitation is given by one vortex sitting at $\boldsymbol{x}=0$, whose wave function is (25) with $\omega^{\uparrow}(z)=\sqrt{\rho_{0}} z$ and $\omega^{\uparrow}(z)=0$. The topological charge is concentrated at the vortex center, $Q^{V}(\boldsymbol{x})=\delta(\boldsymbol{x})$. An approximate solution of the soliton equation (27) reads

$$
\varrho_{\mathrm{vor}}(\boldsymbol{x}) \simeq-\rho_{0}\left(1+\frac{\sqrt{2} r}{\ell_{B}}-\frac{r^{2}}{3 \ell_{B}^{2}}\right) e^{-\sqrt{2} r / \ell_{B}} .
$$

A vortex is a hole made in the condensate of composite bosons.

\subsection{Skyrmion Excitations}

The $\mathrm{SU}(2)$ excitation is generated on the ground state when $\chi(\boldsymbol{x})=$ constant and $\partial_{k} n^{\alpha}(\boldsymbol{x}) \neq$ 0 in (26). The complex-projective field is solved as

$$
n^{\alpha}(\boldsymbol{x})=\frac{\omega^{\alpha}(z)}{\sqrt{\left|\omega^{\uparrow}(z)\right|^{2}+\left|\omega^{\downarrow}(z)\right|^{2}}},
$$

with arbitrary analytic functions $\omega^{\alpha}(z)$. It is known to describe skyrmions [18. We call them spin-skyrmions since they are associated with the spin coherence. The simplest excitation is given by one skyrmion with scale $\kappa$ sitting at $\boldsymbol{x}=0$, whose wave function is

$$
\mathfrak{S}_{\text {sky }}^{\text {spin }}[\boldsymbol{x}]=\prod_{r}\left(\begin{array}{c}
z_{r} \\
\kappa / 2
\end{array}\right)^{\text {spin }} \mathfrak{S}_{\mathrm{LN}}[\boldsymbol{x}] .
$$


The skyrmion is reduced to the vortex in the limit $\kappa \rightarrow 0$. The scale $\kappa$ is to be fixed dynamically. The normalized spin (21) is calculated from this wave function as

$$
s_{\mathrm{sky}}^{x}=\sqrt{1-\left(s^{z}\right)^{2}} \cos \theta, \quad s_{\mathrm{sky}}^{y}=-\sqrt{1-\left(s^{z}\right)^{2}} \sin \theta, \quad s_{\mathrm{sky}}^{z}=\frac{r^{2}-\left(\ell_{B} \kappa\right)^{2}}{r^{2}+\left(\ell_{B} \kappa\right)^{2}} .
$$

The spin flips at the skyrmion center, $s=(0,0,-1)$ at $r=0$, while the spin-up-polarized ground state is approached away from it, $s=(0,0,1)$ for $r \gg \kappa \ell_{B}$.

The soliton equation (27) follows together with the topological charge density,

$$
Q_{0}(\boldsymbol{x})=Q_{0}^{P}(\boldsymbol{x}) \equiv-\frac{i}{2 \pi} \sum_{\alpha} \varepsilon_{j k} \partial_{j}\left(n^{\alpha *} \partial_{k} n^{\alpha}\right) .
$$

The topological charge is shown 18 to be identical with the Pontryagin number whose current density is

$$
Q_{\mu}^{P}(\boldsymbol{x})=\frac{1}{8 \pi} \varepsilon_{a b c} \varepsilon_{\mu \nu \lambda} s^{a} \partial^{\nu} s^{b} \partial^{\lambda} s^{c}
$$

Approximate solutions of the soliton equation (27) are constructed in the two limits, the large skyrmion limit $(\kappa \gg 1)$ and the small skyrmion limit $(\kappa \ll 1)$. First, in the large limit we can solve (27) iteratively, where the first order term is

$$
\varrho_{\mathrm{sky}}(\boldsymbol{x}) \simeq-\nu Q_{0}^{P}(\boldsymbol{x})=-\frac{\nu}{\pi} \frac{\left(\kappa \ell_{B}\right)^{2}}{\left[r^{2}+\left(\kappa \ell_{B}\right)^{2}\right]^{2}} .
$$

This agrees with the formula due to Sondhi et al. [10]. However, in the small limit the topological charge $Q_{0}^{P}(\boldsymbol{x})$ is localized within the core. Indeed, we have $Q_{0}^{P}(\boldsymbol{x}) \rightarrow \delta(\boldsymbol{x})$ as $\kappa \rightarrow 0$ in (36), with which the solution of the soliton equation (27) is approximated by the vortex configuration $\varrho_{\text {vor }}(\boldsymbol{x})$ in $(30)$. This is what we have expected since the skyrmion wave function is reduced to the vortex wave function in the limit $\kappa \rightarrow 0$, where there is no distinction between the $\mathrm{U}(1)$ and $\mathrm{SU}(2)$ excitations. Since the vortex may be considered as a small skyrmion limit, we do not make a clear distinction between them in what follows.

\section{Bilayer QH Systems}

We proceed to analyze bilayer $\mathrm{QH}$ systems. In this section we freeze the spin degree of freedom. We are interested in the so-called Halperin $(m, m, m)$ phase [20], or the interlayercoherent phase, in which an interlayer quantum coherence develops spontaneously [12, 13. We denote the electron field at the layer $\alpha(=1,2)$ by $\psi_{\alpha}$. We call the layer $\alpha=1$ the front layer and the layer $\alpha=2$ the back layer. It is convenient to introduce the pseudospin $\mathrm{SU}(2)$ structure by considering a two-component electron field $\Psi(\boldsymbol{x})$ made of $\psi_{1}$ and $\psi_{2}$. We use the pseudospin index $a=1,2,3$ and define the pseudospin density and the normalized pseudospin field by (20) and (21), respectively. In the interlayer-coherent phase, the dressed CB field 
$\Phi(\boldsymbol{x})$ is defined precisely as in the monolayer QH ferromagnet. The kinetic Hamiltonian of composite bosons is formally identical to that of the monolayer $\mathrm{QH}$ ferromagnet, and given by (18). Consequently, the LLL condition is identical to (19), and the Hilbert space made of the states in the lowest Landau level is identical to that of the monolayer QH ferromagnet. We make a full use of this mathematical identity [13] to investigate the interlayer-coherent phase.

The Coulomb interaction is summarized into the two terms [13],

$$
\begin{aligned}
& H_{C}^{+}=\frac{1}{2} \int d^{2} x d^{2} x V^{+}(\boldsymbol{x}-\boldsymbol{y}) \varrho(\boldsymbol{x}) \varrho(\boldsymbol{y}), \\
& H_{C}^{-}=2 \int d^{2} x d^{2} x V^{-}(\boldsymbol{x}-\boldsymbol{y}) \Delta S^{3}(\boldsymbol{x}) \Delta S^{3}(\boldsymbol{y}),
\end{aligned}
$$

where $V^{ \pm}(\boldsymbol{x})=\left(e^{2} / 2 \varepsilon\right)\left(|\boldsymbol{x}|^{-1} \pm\left(|\boldsymbol{x}|^{2}+d^{2}\right)^{-1 / 2}\right)$ with the interlayer separation $d$ : $\varrho(\boldsymbol{x})$ is the deviation of the total electron density. The Coulomb energy $H_{C}^{+}$is the driving force to realize the $\mathrm{QH}$ system. The term $H_{C}^{-}$describes the capacitive charging energy between the two layers. The deviation of the pseudospin density from the ground-state value is

$$
\Delta S^{a}(\boldsymbol{x}) \equiv \frac{1}{2}\left[\rho(\boldsymbol{x}) s^{a}(\boldsymbol{x})-\rho_{0} s_{0}^{a}\right],
$$

where $s^{a}(\boldsymbol{x})$ is the normalized pseudospin and $s_{0}^{a}$ is its average value in the ground state: See (43). On the other hand, the tunneling term is given by

$$
H_{T}=-\Delta_{\mathrm{SAS}} \int d^{2} x \Delta S^{1}(\boldsymbol{x})
$$

The tunneling term produces an energy gap $\Delta_{\text {SAS }}$ between the symmetric and antisymmetric states. The pseudospin (interlayer) coherence develops provided the capacitance term $H_{C}^{-}$ and the tunneling term $H_{T}$ are reasonably small.

There is one additional degree of freedom in the bilayer system. By applying bias voltages to the two layers, we can freely control the electron density $\rho_{0}^{\alpha}$ in each layer [16]. Namely, the direction of the pseudospin polarization is controllable [13]. Accordingly, the ground state wave function is given by

$$
\mathfrak{S}_{\mathrm{g}}^{\mathrm{ppin}}[\boldsymbol{x}]=\prod_{r}\left(\begin{array}{l}
\sqrt{1+\sigma_{0}} \\
\sqrt{1-\sigma_{0}}
\end{array}\right)_{r}^{\text {ppin }} \mathfrak{S}_{\mathrm{LN}}[\boldsymbol{x}],
$$

where the pseudospinor is common to all electrons and the parameter $\sigma_{0}$ is a real constant $\left(\left|\sigma_{0}\right| \leq 1\right)$; the index ppin denotes the pseudospin. Note that the ground state (41) has been chosen so as to minimize the tunneling energy. The density ratio between the two layers is

$$
\frac{\rho_{0}^{1}}{\rho_{0}^{2}}=\frac{1+\sigma_{0}}{1-\sigma_{0}} .
$$


The normalized pseudospin is calculated from the wave function (41) as

$$
s_{0}^{1}(\boldsymbol{x})=\sqrt{1-\sigma_{0}^{2}}, \quad s_{0}^{2}(\boldsymbol{x})=0, \quad s_{0}^{3}(\boldsymbol{x})=\sigma_{0} .
$$

The balanced configuration is realized when $\sigma_{0}=0$, where the symmetric state is the ground state and described by (41) with $\sigma_{0}=0$.

We analyze charged excitations on the ground state (41). The easiest way is to use a mapping between the bilayer pseudospin state and the monolayer spin state [13]. The mapping is established by the matrix,

$$
T_{\sigma}=\frac{1}{\sqrt{2}}\left(\begin{array}{cc}
\sqrt{1+\sigma_{0}} & \sqrt{1-\sigma_{0}} \\
\sqrt{1-\sigma_{0}} & -\sqrt{1+\sigma_{0}}
\end{array}\right) .
$$

It is easy to see that $T_{\sigma} \mathfrak{S}_{\mathrm{g}}^{\text {ppin }}=\mathfrak{S}_{\mathrm{g}}^{\text {spin }}$, where $\mathfrak{S}_{\mathrm{g}}^{\text {ppin }}$ is the ground state (41) in the unbalanced bilayer system and $\mathfrak{S}_{g}^{\text {spin }}$ is the ground state (23) in the monolayer QH ferromagnet. All excitations are mapped by this transformation between the two systems. Consequently, the skyrmion excitation on the ground state (41) is obtained from that on (223) as $\mathfrak{S}_{\text {sky }}^{\text {ppin }}=T_{\sigma}^{\dagger} \mathfrak{S}_{\text {sky }}^{\text {spin }}$ with (32), or

$$
\mathfrak{S}_{\text {sky }}^{\text {ppin }}=\prod_{r}\left(\begin{array}{c}
z_{r} \sqrt{1+\sigma_{0}}+(\kappa / 2) \sqrt{1-\sigma_{0}} \\
z_{r} \sqrt{1-\sigma_{0}}-(\kappa / 2) \sqrt{1+\sigma_{0}}
\end{array}\right)^{\text {ppin }} \mathfrak{S}_{\mathrm{LN}}[\boldsymbol{x}] .
$$

This is the pseudospin-skyrmion associated with the pseudospin coherence. The normalized pseudospin (21) is easily calculable from this wave function with the aid of (31). It turns out that one skyrmion excitation consists of two parts, one on the front layer and the other on the back layer.

\section{Bilayer QH Ferromagnets}

We finally analyze the bilayer QH system with spins included. The lowest Landau level contains four energy levels corresponding to the two layers and the two spin states. In the balanced configuration the ground state is the symmetric and spin-up state, which is separated by the Zeeman gap $g^{*} \mu_{B} B$ and/or the tunneling gap $\Delta_{\text {SAS }}$ from the one-particle excited states. In the unbalanced configuration at $\nu=1 / m$ the $\mathrm{QH}$ state is given by

$$
\mathfrak{S}[\boldsymbol{x}]=\prod_{r}\left(\begin{array}{l}
1 \\
0
\end{array}\right)_{r}^{\mathrm{spin}}\left(\begin{array}{l}
\sqrt{1+\sigma_{0}} \\
\sqrt{1-\sigma_{0}}
\end{array}\right)_{r}^{\mathrm{ppin}} \mathfrak{S}_{\mathrm{LN}}[\boldsymbol{x}]
$$

as a tensor product of the spinor and the pseudospinor. When both of the interactions are much smaller than the Coulomb energy, two kinds of quantum coherences develop spontaneously upon this ground state.

We consider the filling factor $\nu=1$ and $\nu=2$. There are two cases. (A) When the Zeeman gap is smaller than the tunneling gap, spin-skyrmions are excited at $\nu=1$ while 
pseudospin-skyrmions are excited at $\nu=2$. (B) When the Zeeman gap is larger than the tunneling gap, pseudospin-skyrmions are excited at $\nu=1$ while spin-skyrmions are excited at $\nu=2$.

We are interested in the activation energy $\Delta_{\text {act }}\left(\sigma_{0}\right)$ as a function of the density imbalance parameter $\sigma_{0}$. Though $\Delta_{\text {act }}\left(\sigma_{0}\right)$ is sensitive to impurities in samples, the difference $\Delta_{\text {act }}\left(\sigma_{0}\right)-$ $\Delta_{\text {act }}(0)$ is not so. The excitation energy of the spin-skyrmion depends on $\sigma_{0}$ only through the capacitance term (38), but that of the pseudospin-skyrmion depends also on the tunneling term (40). The capacitive charging energy arises since one skyrmion consists of two charge excitations, one in the front layer $\left(q_{e}^{1}\right)$ and the other on the back layer $\left(q_{e}^{2}\right)$. It carries the total electric charge (28) with $q_{e}=q_{e}^{1}+q_{e}^{2}=\nu e$.

We analyze spin-skyrmions. Though the normalized pseudospin (43) is not affected by the spin-skyrmion excitation, it induces a deviation of the pseudospin (39),

$$
\left\langle\Delta S^{3}(\boldsymbol{x})\right\rangle \equiv \frac{1}{2}\left\langle\varrho_{\text {sky }}(\boldsymbol{x})\right\rangle s_{0}^{3}=\frac{\sigma_{0}}{2} \varrho_{\text {sky }}(\boldsymbol{x}) .
$$

The charge difference is

$$
q_{e}^{1}-q_{e}^{2}=-2 e \int d^{2} x\left\langle\Delta S^{3}(\boldsymbol{x})\right\rangle=\nu \sigma_{0} e
$$

independently of the details of excitations. Hence, the charge on the front layer is $q_{e}^{1}=$ $\frac{1}{2} \nu e\left(1+\sigma_{0}\right)$, while that on the back layer is $q_{e}^{2}=\frac{1}{2} \nu e\left(1-\sigma_{0}\right)$. The ratio $q_{e}^{1} / q_{e}^{2}$ is the same as the ratio (42) of the electron densities on the two layers, as is expected. The capacitive charging energy (38) is estimated as

$$
\left\langle H_{C}^{-}\right\rangle=\alpha_{C} \frac{e^{2} \sigma_{0}^{2}}{\varepsilon \ell_{B}}
$$

with a numerical constant $\alpha_{C}$ at a fixed value of $d / \ell_{B}$ (the ratio of the interlayer distance $d$ and the magnetic length $\ell_{B}$ ). Its value depends on the details of the excitation. The pseudospin-skyrmion is similarly analyzed, and the same formula as (49) is obtained for its charging energy.

We have compared the above results with the experimental data taken from Ref. 16], where $g^{*} \mu_{B} B / \Delta_{\text {SAS }} \simeq 1 / 4$ at $B=5$ Tesla. See Fig. $\square$. At $\nu=1$ we have fitted the activation-energy data by assuming a spin-skyrmion excitation with size $\kappa=2$, where the relevant parameters are $d=231 \AA$ and $\ell_{B}=120 \AA$. At $\nu=2$ we have fitted the data by assuming a pseudospin-skyrmion excitation with size $\kappa=1.65$ and charge $2 e$, where the relevant parameters are $d=231 \AA, \ell_{B}=228 \AA$ and $\Delta_{\text {SAS }}=6 \mathrm{~K}$. The agreements are quite good, as would imply that the present picture of the bilayer QH ferromagnet is basically correct. Detailed analysis will be published in a separate paper [21]. 


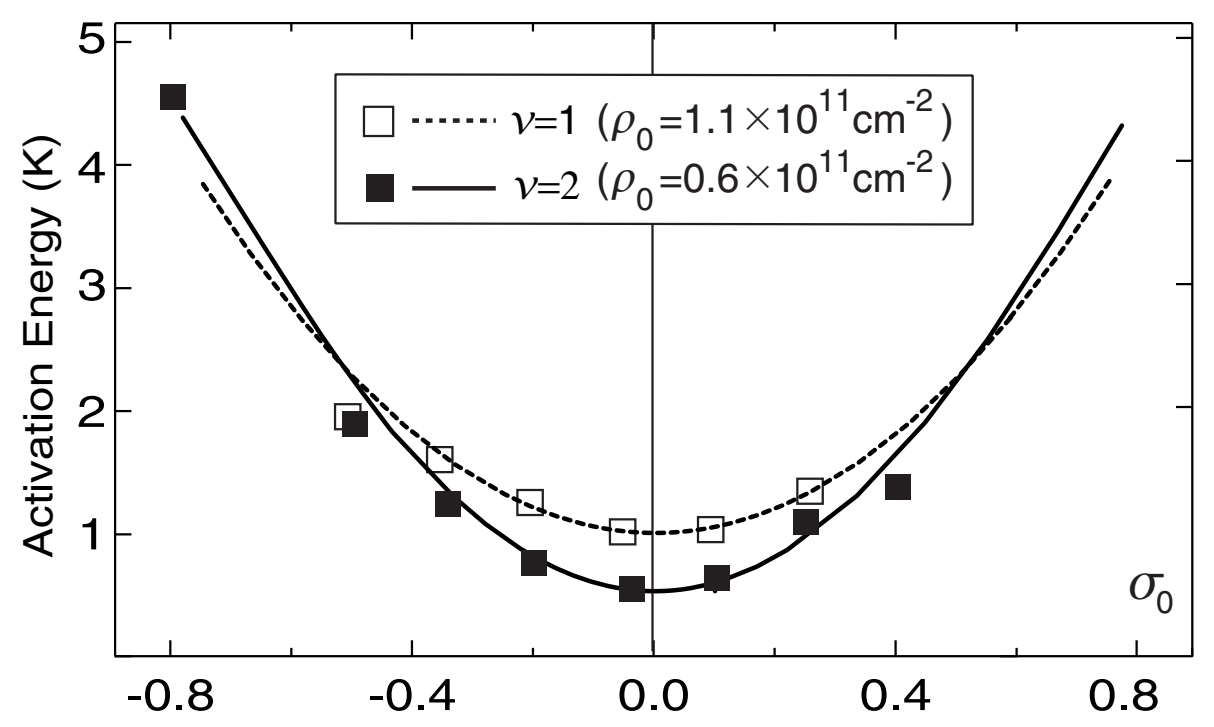

Figure 1: The excitation energy (in $K$ ) of one skyrmion is calculated as a function of the imbalance parameter $\sigma_{0}$, and compared with the experimental data taken from Ref.[16]. We have adjusted the value at the balanced point $\left(\sigma_{0}=0\right)$ to the experimental one.

\section{Discussions}

We have studied both the monolayer and bilayer QH ferromagnets based on the improved CB theory. We have investigated soliton excitations confined to the lowest Landau level. The semiclassical approximation is powerful when the $\mathrm{CB}$ wave function $\mathfrak{S}_{\varphi}$ is factorizable. This allows us to analyze quasiholes successfully. It is quite difficult to make a similar analysis of quasielectrons, for which $\mathfrak{S}_{\varphi}$ is not factorizable. In comparing our theoretical results with experimental data, we have made a physical assumption that the excitation energy of one quasielectron is approximately the same as that of one quasihole. In order to discuss quasielectrons, it would be necessary to make the LLL projection after exciting them. We hope to discuss on this point in a future paper.

I would like to thank K. Sasaki and A. Sawada for various discussions on the subject. A partial support is acknowledged from a Grant-in-Aid for the Scientific Research from the Ministry of Education, Science, Sports and Culture. 


\section{References}

[1] The Quantum Hall Effect, edited by S. Girvin and R. Prange (Springer-Verlag, New York, 1990), 2nd ed.

[2] S.M. Girvin and A.H. MacDonald, Phys. Rev. Lett. 58, (1987) 1252; S.C. Zhang, T.H. Hansen and S. Kivelson, Phys. Rev. Lett. 62, (1989) 82; Z.F. Ezawa and A. Iwazaki, Phys. Rev. B 43, (1991) 2637; Z.F. Ezawa, M. Hotta and A. Iwazaki, Phys. Rev. B 46, (1992) 7765; S.C. Zhang, Int. J. Mod. Phys. B 6, (1992) 25.

[3] S.M. Girvin, in Ref.[1], Chap 10.

[4] N. Read, Phys. Rev. Lett. 62, (1989) 86.

[5] R. Rajaraman and S.L. Sondhi, Int. J. Mod. Phys. B 10, (1996) 793.

[6] J.K. Jain, Phys. Rev. Lett. 63, (1989) 199.

[7] A. Lopez and E. Fradkin, Phys. Rev. B 44, (1991) 5246; V. Kalmeyer and S.C. Zhang, Phys. Rev. B 46, (1992) 9889; B.I. Halperin, P.A. Lee and N. Read, Phys. Rev. B 47, (1993) 7312 .

[8] Z.F. Ezawa, Physica B 249-251, (1998) 841

[9] R. Shankar and G. Murthy, Phys. Rev. Lett. 79, (1997) 4437; D.H. Lee, condmat/9709233; V. Pasquier and F.D.M. Haldane, cond-mat/9712169.

[10] S.L. Sondhi, A. Karlhede, S. Kivelson and E.H. Rezayi, Phys. Rev. B 47, (1993) 16419; D.H. Lee and C.L. Kane, Phys. Rev. Lett. 64, (1990) 1313.

[11] S.E. Barrett, G. Dabbagh, L.N. Pfeiffer, K.W. West and R. Tycko, Phys. Rev. Lett. 74, (1995) 5112; A. Schmeller, J.P. Eisenstein, L.N. Pfeiffer and K.W. West, Phys. Rev. Lett. 75, (1995) 4290; E.H. Aifer, B.B. Goldberg and D.A. Broido, Phys. Rev. Lett. 76, (1996) 680; D.K. Maude, M. Potemski, J.C. Portal, M. Henini, L. Eaves, G. Hill and M.A. Pate, Phys. Rev. Lett. 76, (1996) 4604.

[12] Z.F. Ezawa and A. Iwazaki, Int. J. Mod. Phys. B 6, (1992) 3205; Phys. Rev. B 47, (1993) 7295; Phys. Rev. B 48, (1993) 15189;

[13] Z.F. Ezawa, Phys. Lett. A 229, (1997) 392; Z.F. Ezawa, Phys. Rev. B 55, (1997) 7771.

[14] K. Moon, H. Mori, K. Yang, S.M. Girvin, A.H. MacDonald, L. Zheng, D. Yoshioka and S.C. Zhang, Phys. Rev. B 51, (1995) 5138. 
[15] S.Q. Murphy, J.P. Eisenstein, G.S. Boebinger, L.N. Pfeiffer and K.W. West, Phys. Rev. Lett. 72, (1994) 728.

[16] A. Sawada, Z.F. Ezawa, H. Ohno, Y. Horikoshi, Y. Ohno, S. Kishimoto, F. Matsukura, M. Yasumoto, and A. Urayama, Phys. Rev. Lett. 80, (1998) 4534.

[17] R.B. Laughlin, Phys. Rev. Lett. 50, (1983) 1395.

[18] A. D’Adda, A. Luscher and P. DiVecchia, Nucl. Phys. B146, (1978) 63.

[19] L. Saminadayar, D.C. Glattli, Y. Jin and B. Etienne, Phys. Rev. Lett. 79, (1997) 2526;

R. de-Picciotto, M. Reznikov, M. Heiblum, V. Umansky, G. Bunin andd D. Mahalu, Nature 386, (1997) 162.

[20] B.I. Halperin, Helv. Phys. Acta 56, (1983) 75.

[21] Z.F. Ezawa, K. Sasaki and A. Sawada, in preparation. 